\title{
A hybrid artificial neural network-differential dynamic programming approach for short-term hydro scheduling
}

\author{
Ruey-Hsun Liang, Yuan-Yih Hsu \\ Department of Electrical Engineering, National Taiwan University, Taipei, Taiwan
}

Received 10 October 1994

\begin{abstract}
In this paper, a hybrid artificial neural network-differential dynamic programming (ANN-DDP) method for the scheduling of short-term hydro generation is developed. The purpose of short-term hydro scheduling is to find the optimal amounts of generated powers for the hydro units in the system for the next $N(N=24$ in this work) hours in the future. In the proposed method, the DDP procedures are performed offline on historical load data. The results are compiled and valuable information is obtained by using ANN algorithms. The DDP algorithm is then performed online according to the obtained information to give the hydro generation schedule for the forecasted load. Two types of ANN algorithm, the supervised learning neural network by Rumelhart et al. and the unsupervised learning neural network by Kohonen, are employed and compared in this paper. The effectiveness of the proposed approach is demonstrated by the short-term hydro scheduling of Taiwan power system which consists of ten hydro plants. It is concluded from the results that the proposed approach can significantly reduce the execution time of the conventional differential dynamic programming algorithm which is required to reach proper hydro generation schedules.
\end{abstract}

Keywords: Hydro scheduling; Neural networks; Differential dynamic programming

\section{Introduction}

The major objective of hydro generation scheduling in a power system is to minimize the total fuel cost of thermal units by utilizing the limited water resource for the next $N$ ( $N=24$ in this work) hours in the future. It is a typical constrained optimization problem in which the total operating cost over the study period is minimized subject to load and system constraints. In the literature, numerous approaches [1-7] have been reported to solve this problem. Among them, the dynamic programming (DP) approach has gained much popularity because a great number of hydroelectric generation scheduling problems can be formulated as a dynamic optimization problem. The differential dynamic programming (DDP) algorithm is a promising DP based solution method for large-scale problems [7]. Quite promising results in terms of fuel cost savings have been reached in most of the works reported so far. However, most methods require too much computation time to get the desired solution. Since the daily load pattern of a certain day is similar to those for days of the same type, the efficiency of the aforementioned methods can be significantly improved if operating experiences and offline analysis of historical records are utilized [8].

For a complicated constrained optimization problem whose feasible solution space is large, such as the hydro scheduling problem considered in this work, it usually takes a long time to reach the desired solution. Systematic search algorithms, such as differential dynamic programming [9], improve solution efficiency by following search strategies in which prescheduled feasible solutions are used. Therefore, how to improve the efficiency of the search procedures by using available information on the search space to give an efficient initial control trajectory is the major concern in this paper.

Two kinds of artificial neural network (ANN) algorithm, the supervised learning network by Rumelhart et al. $[10,11]$ and the unsupervised learning network by Kohonen [12], are developed to play the role of trajectory search assistant. In the past few years, efforts have been placed on the application of ANNs to power 
systems. Many interesting applications of neural nets in the power field have been reported, such as load forecasting [13], power system stabilizer design [14], capacitor control [15], unit commitment [8,16], torsional oscillation analysis [17], and security assessment [1820]. A major advantage of the ANN approach is that the domain knowledge is distributed in the neurons and information processing is carried out in a parallel-distributed manner. Therefore, it is rather efficient for the ANN to perform the desired computations.

In this paper, to speed up short-term hydro generation scheduling, we propose a hybrid artificial neural network-differential dynamic programming (ANNDDP) approach. The proposed method is essentially divided into two parts, offline training and online scheduling. In the training phase, we collect historical load records and the corresponding optimal hydro schedules which have been reached by using the DDP approach and use the load-schedule patterns to train the ANN offline. Then, in online applications, we employ the trained ANN to obtain the preliminary schedule for a given forecasted load. This preliminary schedule is then used as an initial control trajectory for the DDP algorithm. Thus, the ANN-DDP approach can significantly reduce the execution time of the DDP algorithm required to reach proper hydro schedules.

In the next section, the hydroelectric generation scheduling problem and the DDP algorithm with its applications to the scheduling of hydro generation are stated. The proposed hybrid ANN-DDP method is explained in Section 3. In Section 4, details of the two ANN algorithms, supervised learning neural net and unsupervised learning neural net, used in this study are described. Finally, the effectiveness of the proposed ANN-DDP approach is demonstrated by the hydro scheduling of Taiwan power system which consists of four Ta-Chia River cascaded plants, three Cho-Shui River plants, and three hydraulically independent plants. It is concluded from the results that proper hydro generation schedules can be obtained in a very efficient manner by using the proposed ANN-DDP approach.

\section{Hydro generation scheduling by differential dynamic programming}

\subsection{Problem formulation}

Scheduling hydro generation is well known to be coupled with its thermal counterpart. Under present Taiwan Power Company (TPC) system operating conditions, short-term commitment changes are not allowed for most of the base-load and medium-load thermal units. The scheduling of hourly thermal generation is thus reduced to an economic dispatch problem, in which the thermal unit commitment aspect can be ignored. To meet the spinning reserve requirement, TPC keeps a certain percentage of the available thermal capacities (committed units and all the peaking units) as part of the spinning reserve. For a given load level, the remaining spinning reserve capacity that should be contributed by the hydro units can then be determined. According to the aforementioned operation of the TPC system, the coupling between hydro and thermal generation schedulings is only through the constraint that the total generation should meet the system load.

We decouple the hydro scheduling from the thermal part by first establishing the generation cost function of meeting the system load by purely thermal generation. For each given load level, the lambda-iteration method is performed to solve the economic dispatch over the set of available units [2] and to evaluate the thermal generation cost in order to meet the load demand. In other words, we aggregate all the available thermal units into one equivalent unit and construct its generation cost function. Then, in hydro scheduling, we try to find the best way of substituting hydro for thermal energy based on this function so that the system generation cost is minimized. (In TPC's hydro system, there is no significant delay relative to the one-hour time increment for water to flow from one reservoir to its immediate downstream neighbor.) To do this, the study period (one day for the present work) is divided into $N$ stages ( $N=24$ in the present case) and the hydro scheduling problem is then formulated as follows.

Minimize $C=\sum_{t=1}^{24} \operatorname{COST}_{t}\left(\mathrm{GTHERMAL}_{t}\right)$

subject to

(1) the generation-load balance equations

GTHERMAL $_{t}+\sum_{i} P_{i}\left(X_{i t}\right)=L_{t} \quad t=1, \ldots, 24$

(2) the water balance equations

$$
\begin{array}{r}
Y_{i, t+1}=Y_{i t}+\sum_{j \in \mathrm{N}_{i}} X_{j t}-X_{i t}+\sum_{i \in \mathrm{N}_{i}} S_{l t}-S_{i t}+R_{i t} \\
i=1, \ldots, I ; \quad t=1, \ldots, 24
\end{array}
$$

(3) bounds on water releases

$$
\begin{aligned}
& X_{i, \min } \leqslant X_{i t} \leqslant X_{i, \max } \text { and } S_{i, \min } \leqslant S_{i t} \leqslant S_{i, \max } \\
& i=1, \ldots, I
\end{aligned}
$$

(4) bounds on reservoir storage

$\mathrm{Y}_{i, \min } \leqslant Y_{i t} \leqslant Y_{i, \max } \quad i=1, \ldots, I$

(5) the hydro spinning reserve requirement

$\sum_{i}\left[P_{i}\left(X_{i, \max }\right)-P_{i}\left(X_{i t}\right)\right] \geqslant \mathrm{SR}_{t} \quad t=1, \ldots, 24$

with the following nomenclature: 


\begin{tabular}{|c|c|}
\hline$C$ & $\begin{array}{l}\text { system generation cost over study pe- } \\
\text { riod }\end{array}$ \\
\hline $\operatorname{CosT}_{r}(\cdot)$ & $\begin{array}{l}\text { generation cost function at hour } t \\
\text { which is approximated by a second- } \\
\text { order polynomial }\end{array}$ \\
\hline GTHERMAL & $\begin{array}{l}\text { total generation from thermal units at } \\
\text { hour } t\end{array}$ \\
\hline$I$ & $\begin{array}{l}\text { number of reservoirs ( } I=10 \text { in present } \\
\text { work) }\end{array}$ \\
\hline$L_{t}$ & system load at hour $t$ \\
\hline$N_{i}$ & $\begin{array}{l}\text { set of reservoirs immediately upstream } \\
\text { of reservoir } i\end{array}$ \\
\hline$P_{i}(\cdot)$ & $\begin{array}{l}\text { water-to-energy conversion function } \\
\text { of power plant associated with reser- } \\
\text { voir }\end{array}$ \\
\hline$R_{i t}$ & $\begin{array}{l}\text { volume of natural inflow to reservoir } i \\
\text { during hour } t\end{array}$ \\
\hline$S_{i t}$ & spillage from reservoir $i$ during hour $t$ \\
\hline $\mathrm{SR}_{t}$ & $\begin{array}{l}\text { hydro spinning reserve requirement at } \\
\text { hour } t\end{array}$ \\
\hline$X_{i t}$ & $\begin{array}{l}\text { volume of water released from reser- } \\
\text { voir } i \text { for generation during hour } t\end{array}$ \\
\hline$Y_{i t}$ & $\begin{array}{l}\text { water stored in reservoir } i \text { at beginning } \\
\text { of hour } t\end{array}$ \\
\hline
\end{tabular}

For this hydro generation scheduling problem, we first employ the DDP algorithm, described briefly in the following, to deal with this problem. Then, an ANN DDP approach, explained in Section 3, is proposed to speed up the execution time for the same problem.

\subsection{Hydro scheduling by differential dynamic programming}

The input data for the hydro scheduling program contain $N\left(N=24\right.$ in this study) hourly loads $L_{t}$ $(t=1,2, \ldots, 24)$. Given these hourly loads, our purpose is to determine the amount of water released for hydro generations $X_{i t}$ for each unit $i$ such that the total fuel cost of the thermal units is minimized.

Let $\boldsymbol{U}$ be the input vector which comprises the 24 hourly loads, i.e.

$$
\begin{aligned}
\boldsymbol{U} & =\left[u_{1}, u_{2}, \ldots, u_{24}\right]^{\mathrm{T}} \\
& =\left[L_{1}, L_{2}, \ldots, L_{24}\right]^{\mathrm{T}}
\end{aligned}
$$

Let us also define $Z$ as the output vector which comprises the volume of water released from each reservoir for hydro generation $X_{i t}(i=1,2, \ldots, 10$; $t=1,2, \ldots, 24)$ :

$$
\begin{aligned}
\boldsymbol{Z} & =\left[z_{1}, z_{2}, \ldots, z_{240}\right]^{\mathbf{T}} \\
& =\left[X_{11}, \ldots, X_{10,24}\right]^{\mathbf{T}}
\end{aligned}
$$

To deal with the above hydro generation scheduling problem, we employ the DDP algorithm described briefly in this section. The advantages of the DDP algorithm are that it explicitly exploits system dynamics and it avoids the curse of dimensionality of the DP, but it suffers from computational inefficiency. We propose to improve the efficiency of the DDP algorithm using the hybrid ANN-DDP approach described in the next section.

Because the DDP algorithm is an unconstrained dynamic optimization method, we first relax all the constraints for the hydro scheduling problem described in the above section, except the system dynamics in Eq. (3), by using the multiplier method [9]. Then, the hydro scheduling problem becomes an unconstrained dynamic optimization problem to be solved by the DDP algorithm. It is a successive approximation technique and consists of two basic procedures: approximate backward dynamic programming and successive control construction. The backward procedure takes a quadratic approximation of the objective function and a linear approximation of the system dynamics along a nominal trajectory. Dynamic programming is then applied to the approximate linear and quadratic problem to find control variations. The successive control construction procedure constructs, forward in time, new controls that result in a lower cost by searching in the direction of the control variations obtained in the backward procedure. The nominal trajectory is then updated by the new trajectory. Both procedures are carried out repeatedly till a convergent solution is achieved. Details of the DDP algorithm can be found in Ref. [21].

Two levels, high-level multiplier updating and lowlevel minimization, are alternated iteratively until the controls $X_{i t}^{*}$, which are the water release volumes of each hydro plant at each hour, converge to the optimal solution.

From the previous descriptions of the DDP algorithm, it is noted that, if we can find proper initial controls $X_{i t}^{0}$ which are close to the optimal or nearoptimal controls $X_{i t}^{*}$, the computation time will be significantly reduced. This motivates the development of a hybrid ANN-DDP approach in which an ANN is used to yield a preliminary schedule $X_{i t}$ which is close to the optimal solution.

\section{The proposed hybrid ANN-DDP approach}

The proposed method can be divided into two major parts: offline ANN training and online scheduling by the hybrid ANN-DDP approach. Details of the method are described as follows.

\subsection{Offline ANN training}

The training of artificial neural networks involves three major steps, as shown in Fig. 1. In the first step, 


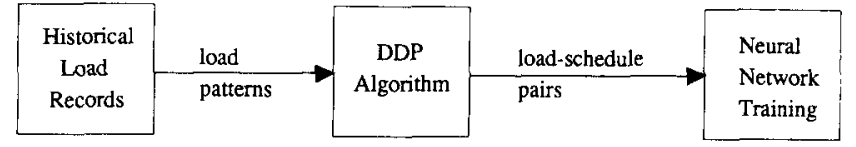

Fig. 1. ANN training procedures.

a total of 92 historical load patterns in the summer of 1990 were collected. Among these load patterns, the load records for the first day of each month are reserved as test patterns. This leaves only 89 patterns as the training patterns. Here, a load pattern is a 24dimensional vector $\boldsymbol{U}=\left[L_{1}, L_{2}, \ldots, L_{24}\right]$, in which $L_{\mathrm{t}}$ is the system load at hour $t$.

In the second step, the DDP algorithm as described in Section 2.2 is employed to generate the corresponding optimal or near-optimal schedules for the 89 load patterns. Thus, we have 89 load-schedule $(\boldsymbol{U}, \boldsymbol{Z})$ pairs.

In the final step, two kinds of neural network, the supervised learning neural network proposed by Rumelhart et al. [10] and the unsupervised learning neural network proposed by Kohonen [12], are trained using the 89 load-schedule $(U, Z)$ pairs at hand. The multilayer feedforward ANN can be trained by using the backpropagation method. Details for the training of the multilayer feedforward ANN will be described in Section 4.1 .

As for the Kohonen self-organizing neural net training, we use the 89 load $(U)$ patterns to separate them into clusters. Details of the clustering ANN will be described in Section 4.2.

\subsection{Online scheduling by using the hybrid $A N N-D D P$ approach}

The proposed hybrid ANN-DDP approach can be divided into two major parts as shown in Fig. 2. In the first part, we try to determine a preliminary schedule by using the ANN. Then, we use the DDP algorithm to generate the final schedule. Details of the method are described as follows.

\subsubsection{Preliminary scheduling by ANNS}

To reach a preliminary schedule we propose to use both Rumelhart's ANN and Kohonen's ANN. In applying Rumelhart's ANN (RANN) the inputs to the ANN are the 24 hourly system loads under study, $U_{f}$,

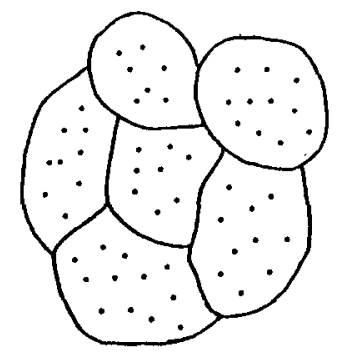

Fig. 3. A clustering of two-dimensional points.

and the outputs contain the preliminary volumes of released water for hydro generation, $X_{i t}(i=1, \ldots, 10$, $t=1, \ldots, 24)$. In the second method, using Kohonen's ANN (KANN), each load pattern is described by a 24-dimensional vector and we try to group the 89 load patterns into clusters. Fig. 3 shows a conceptual figure of a clustering where points of two-dimensional patterns are grouped into clusters according to their geometric similarity.

Now, for the load pattern under study, $\boldsymbol{U}_{\mathrm{f}}$, one can find a group which is most similar to the study load pattern. Let us identify this group as G. Thus, the average of the $Z$ vectors which are associated with the $U$ patterns in group $G$ should contain valuable information with regard to the described schedule for the study pattern $\boldsymbol{U}_{\mathrm{f}}$. Let $\boldsymbol{O}_{\mathrm{f}}$ be this average vector. In other words,

$\boldsymbol{O}_{\mathrm{f}}=\operatorname{average}\left(\boldsymbol{Z}_{m} \mid \boldsymbol{U}_{\mathrm{m}} \in \mathrm{G}\right)$

where $Z_{m}$ is the schedule vector corresponding to load pattern $\boldsymbol{U}_{m}$ in group G. Note that the vector $\boldsymbol{O}_{\mathrm{f}}$ gives the desired preliminary hydro schedules for the day under study. In other words, the hourly water releases for the ten hydro plants are the elements of the vector $\boldsymbol{O}_{\mathrm{f}}$.

\subsubsection{Final scheduling by the $D D P$ algorithm}

Now, with the preliminary volumes of released water $X_{i f}$ for the study load pattern $U_{\mathrm{f}}$ at hand, we can proceed to use the preliminary schedules $X_{i t}$ as the initial controls $X_{i t}^{0}(i=1, \ldots, 10 ; t=1, \ldots, 24)$ of the DDP algorithm in order to reach the final schedule. Since the initial controls $X_{i t}^{0}$ are very close to the optimal or near-optimal controls $X_{i t}^{*}$, the computation time of the DDP algorithm can be significantly reduced.

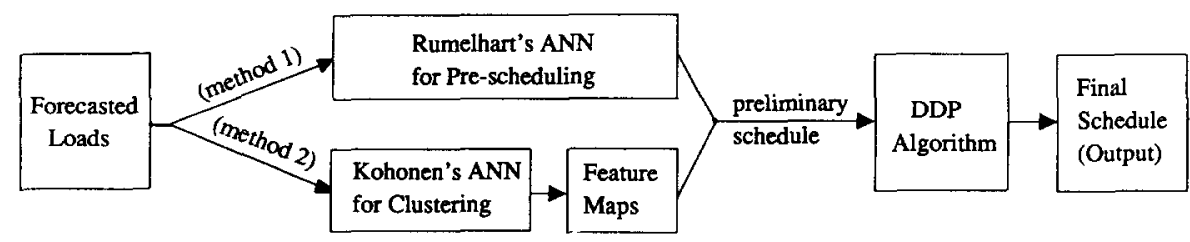

Fig. 2. The hybrid ANN-DDP approach. 


\section{Design of the artificial neural networks}

It was mentioned that we need two kinds of artificial neural network, the supervised learning neural network of Rumelhart et al. [10] and the unsupervised learning neural network of Kohonen [12], in order to reach the preliminary schedule.

\subsection{The multilayer feedforward $A N N$}

The structure of a multilayer feedforward ANN is shown in Fig. 4. The nodes in the input layer receive input signals from the outside world and directly pass the signals to the nodes in the next layer. In this paper, the 24 hourly loads $L_{t}(t=1,2, \ldots, 24)$ are taken as the imputs of the neural network.

The nodes in the output layer provide the desired hydro generation schedule which is characterized by the volumes of water released from the reservoirs, $X_{i t}$ $(i=1,2, \ldots, 10 ; t=1,2, \ldots, 24)$. Therefore, we need 240 output nodes. In addition to the input layer and output layer, we need one or more hidden layers. The nodes in the hidden layer take signals from the nodes in the input layer and send their outputs to the nodes in the next layer when computations within the nodes have been completed. In the present work, only one hidden layer is employed.

For each neuron $i$ in the hidden layer and output layer, the neuron output is given by

$o_{i}=f_{i}\left(\right.$ net $\left._{i}, \theta_{i}\right)=\frac{1}{1+\exp \left[-\left(\text { net }_{i}+\theta_{i}\right)\right]}$

where $\theta_{i}$ is a bias and net ${ }_{i}$ is the input signal to neuron $i$, expressed as

$\operatorname{net}_{i}=\sum_{j} w_{i j} o_{j}$

The summation in Eq. (11) is performed over all nodes $j$ in the preceding layer that are connected with neuron $i$. $w_{i j}$ is the connection weight from neuron $j$ to neuron $i$ and $o_{j}$ is the output of neuron $j$.

The design of an artificial neural network involves two major phases: training and testing. In the training process, we try to determine the connection weights $w_{i j}$ using a set of input-output patterns in the training set. Once the connection weights have been figured out, the performance of the neural network is tested using both the patterns within the training set and the patterns outside the training set. The speed and accuracy of the test results are evaluated in order to decide if modification of the neural network structure (number of hidden layers and hidden nodes per layer) or further training of the neural network is necessary. Once the speed and accuracy of the ANN meet the requirements of the present application, it can be employed to evaluate the hydro generation scheduling in real-time situations. Details of the training process are described as follows.

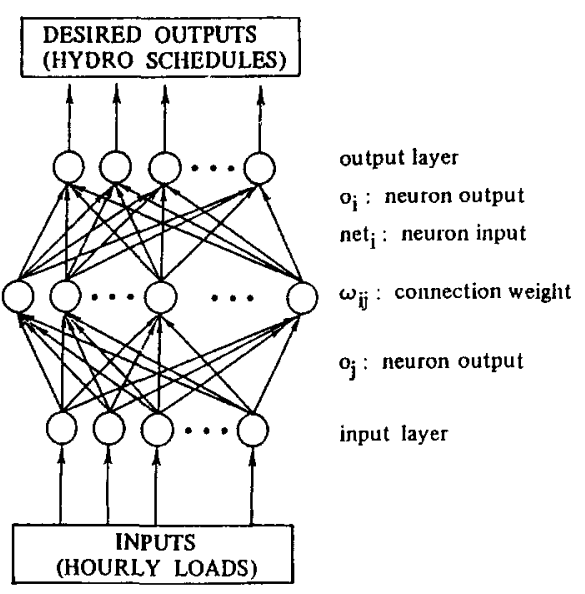

Fig. 4. The structure of a multilayer feedforward ANN.

The first step in neural network training is to compile the training patterns in the training set. The 89 training patterns are obtained by using the DDP technique. With these training patterns at hand, one can proceed to figure out a proper set of connection weights $w_{i j}$ that can best fit the input-output patterns in the training set. A commonly used approach is the generalized delta rule [10], where the sum of the squared errors is minimized, as described below:

$E=\sum_{m=1}^{M}\left(t_{m}-o_{m}\right)^{2}$

where $M$ is the number of output nodes, $t_{m}$ is the desired (target) output for output node $m$ and $o_{m}$ is the computed neuron output. The connection weights $w_{i j}$ are first initialized to random values. After an input output pattern $p$ is presented and the error function $E$ is computed, the connection weights can be updated using the gradient descent method. The connection weights between hidden unit $k$ and output unit $m$ are updated using the following equation [10]:

$\Delta w_{m k}(p)=\eta \delta_{m, p} o_{k, p}+\alpha \Delta w_{m k}(p-1)$

where

$\delta_{m, p}=\left(t_{m, p}-o_{m, p}\right) f_{m}^{\prime}\left(\right.$ net $\left._{m, p}\right)$

$\eta$ and $\alpha$ are the learning rate (step size) and momentum constant, respectively, and $\Delta w_{m k}(p-1)$ is the change in connection weight following the presentation of the previous input -output pattern $(p-1)$. The connection weights from any input unit (or hidden unit) $j$ to a hidden unit $k$ can be updated using similar equations:

$\Delta w_{k j}(p)=\eta \delta_{k, p o j, p}+\alpha \Delta w_{k j}(p-1)$

where

$\delta_{k, p}=f_{k}^{\prime}\left(\right.$ net $\left._{k, p}\right) \sum_{i} \delta_{i, p} w_{i k}$

and $i$ is a node in the layer following that of node $k$. 


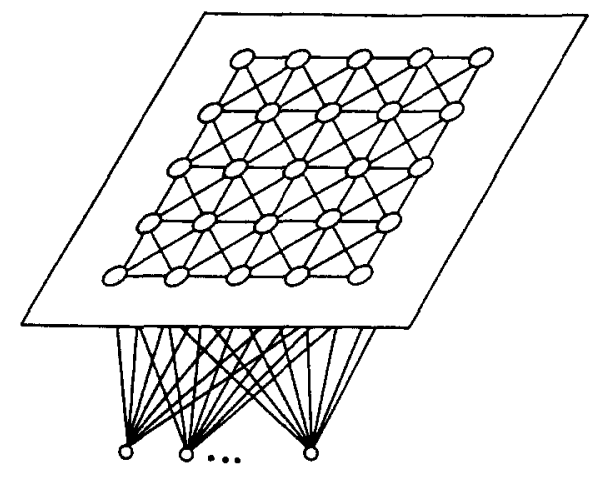

Fig. 5. Kohonen's self-organizing feature map.

After the connection weights have been determined, the ANN can be employed to determine the preliminary hydro schedule for the given load pattern.

\subsection{Kohonen's self-organizing neural net}

The unsupervised learning artificial neural network, Kohonen's self-organizing feature map, was employed to classify the 89 load patterns.

The self-organizing feature map originated from the observation that the fine structure of the cerebral cortex in the brain is self-organized during training. It maps the $N$-dimensional inputs $\left[u_{1}, u_{2}, \ldots, u_{24}\right]^{\mathrm{T}}$ onto the $M$ output nodes $(M=11 \times 11=121$ in our study), as shown in Fig. 5. Each output node is described by a pair of rectangular coordinates. A salient characteristic of Kohonen's net is that the mapping is topologically conservative, which means that 'similar' input vectors in its $N$-dimensional space will be mapped to the nodes within a neighborhood on the two-dimensional grid. Thus, typical applications of Kohonen's net are pattern clustering or pattern classification.

Between every output node and every input node, there is a weighted synaptic connection $w_{i j}$. To each output node, its weight vector $\boldsymbol{W}_{j}$ acts as a templet or exemplar. The degree of activation of each node is directly dependent on the similarity between this templet and the present input pattern.

As an input pattern is presented at the input nodes, the activation value $a_{j}$ of node $j$ is given by the formula

$a_{j}=\frac{1}{\left|\boldsymbol{U}-\boldsymbol{W}_{j}\right|}$

where $\boldsymbol{U}$ is the input pattern vector, which is a 24-hour load record in our study, and $\boldsymbol{W}_{j}=\left[w_{1 j}, w_{2 j}, \ldots, w_{24 j}\right]^{\mathrm{T}}$ is the connection weight vector for output node $j$ $(j=1, \ldots, 121)$.

When an input pattern is presented to the neural net, the neural net computes the activation value for each output node based on the present connection weights. The input pattern is said to be mapped to the output node with the maximum activation value.
Kohonen suggested that the connection weights be initialized to small random values. The approach works well for the cases where the input vectors are widely spread over the whole pattern space. But the input vectors in the present work are restricted to a small portion of the space. Therefore, we propose to set the initial weight vectors to be around the means of these vectors. In other words, we first define

$$
\begin{array}{r}
\boldsymbol{W}_{j}^{\prime}=\text { means of }\left(\boldsymbol{U}_{i}, \quad i=1,2, \ldots, 89\right) \\
\qquad j=1, \ldots, M
\end{array}
$$

Then we perturb $\boldsymbol{W}_{j}^{\prime}$ with a random noise, as follows:

$$
\begin{gathered}
\boldsymbol{W}_{j}=\boldsymbol{W}_{j}^{\prime}+\left[5 r \times \text { variance of }\left(\boldsymbol{U}_{i}, \quad i=1,2, \ldots, 89\right)\right] \\
j=1, \ldots, M
\end{gathered}
$$

where $r$ is a random number uniformly distributed in the range -0.5 to 0.5 .

During the learning process, the output nodes are activated by input vectors. At every presentation of input vectors, the mapped node which has the maximum activation value is identified, and the weight vectors of the mapped node and its neighbors are updated by the formula

$\boldsymbol{W}_{j}(t+1)=\boldsymbol{W}_{j}(t)(1-\eta)+\boldsymbol{U} \eta$

where $\boldsymbol{U}$ is the present input vector and $\eta$ is a step size which decreases during the learning process.

The neighborhood of updating initially covers a wide range and shrinks by iterations. Finally, only the weights of the mapped node are updated.

After enough input pattern vectors have been presented, input patterns with similar features will be mapped to the same output unit or to output units within a small neighborhood.

\section{Example}

To demonstrate the effectiveness of the proposed hybrid ANN-DDP approach, hydroelectric generation scheduling was performed on Taiwan power system which consists of four Ta-Chia River cascaded plants, three Cho-Shui plants (comprising a large pumped storage plant and two cascaded hydro plants) and three hydraulically independent plants. The schematic diagram of the hydro plants along both the Cho-Shui River and the Ta-Chia River is shown in Fig. 6. The hydro system data used for the present work are presented in Table 1. A total of 92 historical load records in the summer of 1990 were collected. Among these load patterns, the load records for the first day of each month were reserved as test patterns. Fig. 7 depicts the system's hourly load curves for the three test load patterns. All our numerical computations were performed on a Sun workstation. 
Table 1

Hydro system data

\begin{tabular}{|c|c|c|c|c|c|}
\hline \multirow[t]{2}{*}{ Reservoir } & \multicolumn{2}{|l|}{ Storage $\left(\mathrm{km}^{3}\right)$} & \multirow[t]{2}{*}{ Plant } & \multicolumn{2}{|c|}{ Water release $\left(\mathrm{m}^{3} / \mathrm{s}\right)$} \\
\hline & Lower bound & Upper bound & & Lower bound & Upper bound \\
\hline Sun-Moon & 13269 & 155685 & Ta-Kuan 2 & -249 & 380 \\
\hline Storage Pond & 1565 & 9407 & Ta-Kuan 1 & 0 & 50 \\
\hline Chu-Kung & 1.6 & 105 & Chu-Kung & 0 & 45 \\
\hline Te-Chi & 89886 & 243120 & Te-Chi & 0 & 217.5 \\
\hline Chin-Shan & 26 & 647 & Chin-Shan & 0 & 174.8 \\
\hline Ku-Kuan & 101 & 6563 & Ku-Kuan & 0 & 133.6 \\
\hline Tien-Lun & 90 & 560 & Tien-Lun & 0 & 68 \\
\hline $\mathrm{Li}-\mathrm{Wu}$ & 0 & 340 & $\mathrm{Li}-\mathrm{Wu}$ & 0 & 36.7 \\
\hline Lung-Chien & 0 & 202 & Lung-Chien & 0 & 13.2 \\
\hline $\mathrm{I}-\mathrm{H} \operatorname{sing}$ & 0 & 1343 & 1-Hsing & 0 & 31.7 \\
\hline
\end{tabular}
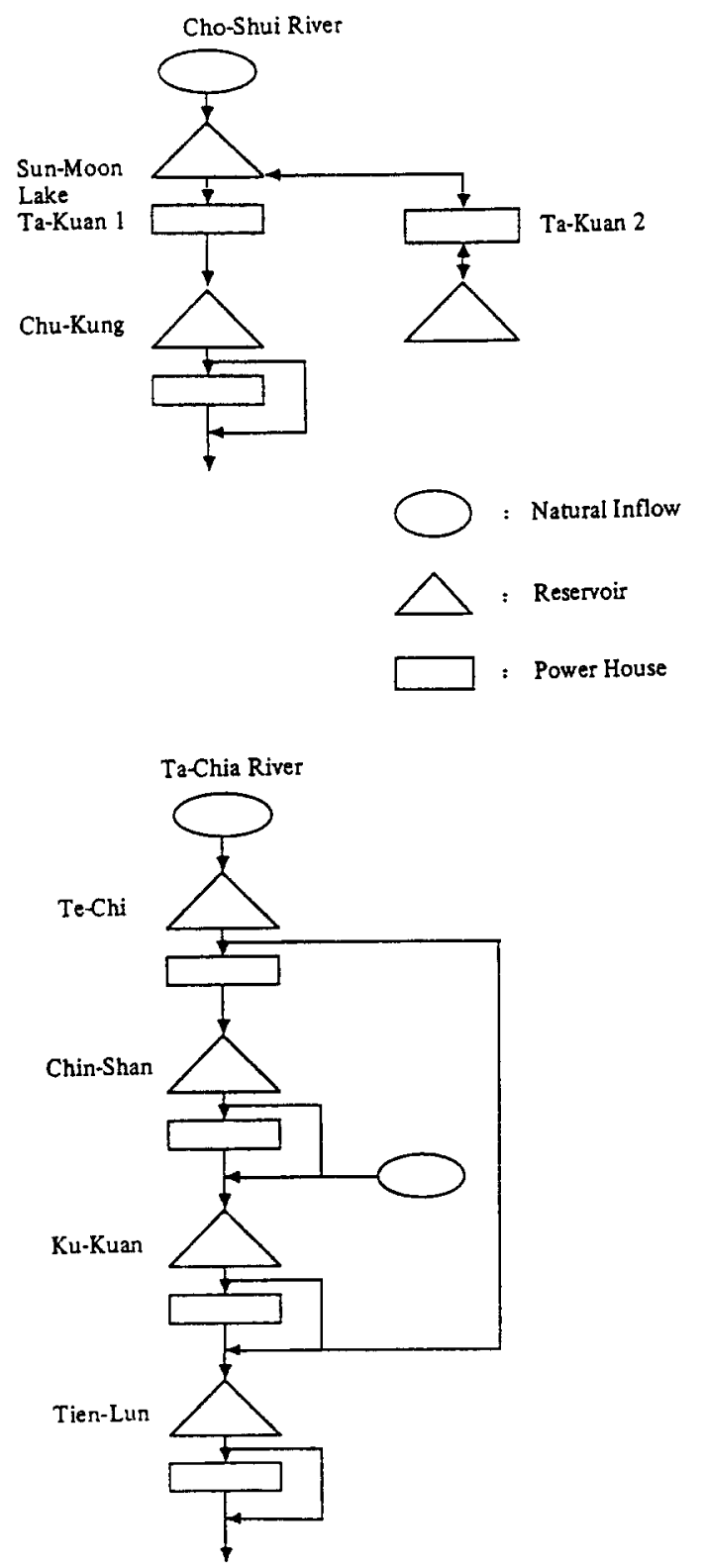

Fig. 6. Schematic diagram of Cho-Shui and Ta-Chia Rivers.

\subsection{Results using the multilayer feedforward ANN}

The multilayer feedforward ANN comprised 24 input nodes which contain 24 hourly load demands, 49 hidden units and 240 output nodes which give the released water schedule for each hydro plant at each hour. In the training process, 89 training patterns were selected in order to determine the connection weights of the neural network. To examine the convergence characteristic of the training algorithm, the root mean square (r.m.s.) errors in the learning process are depicted in Fig. 8. Once the feedforward ANN had been trained, the reserved three load patterns were used to test the proposed algorithm.

After obtaining the preliminary schedules, the DDP algorithm was executed to reach the final solutions. Table 2 compares the results from the DDP algorithm and those from the RANN-DDP approach. Fig. 9 illustrates the water release schedules for one of the ten

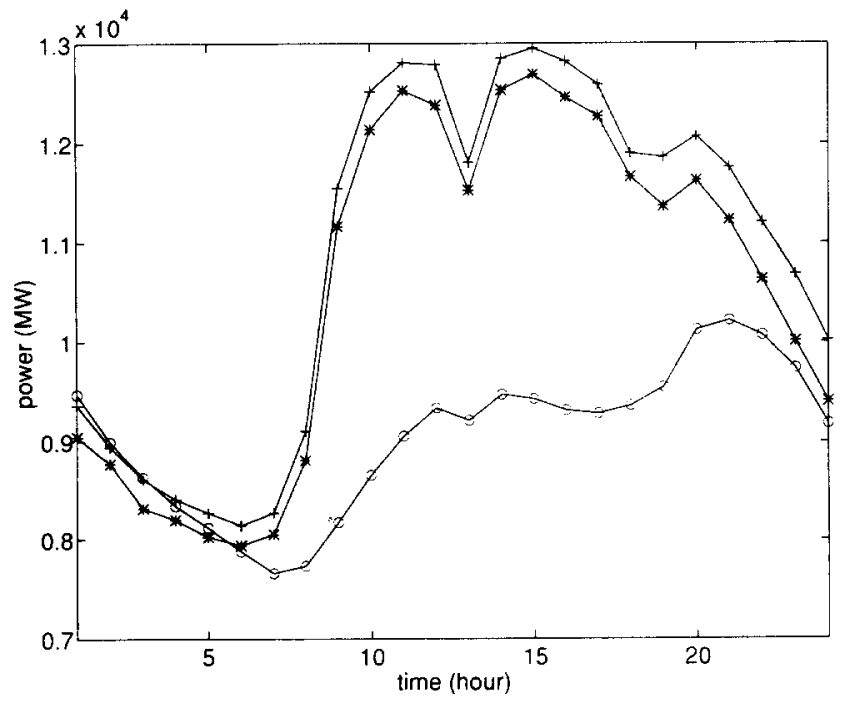

Fig. 7. Daily system load profiles: *, 1 June 1990 (Fri.); 0,1 July 1990 (Sun.); +, 1 Aug. 1990 (Wed.). 
Table 2

Comparison of the results from the DDP approach, the RANN-DDP approach and the KANN-DDP approach

\begin{tabular}{|c|c|c|c|c|c|c|c|c|c|c|c|}
\hline \multirow{2}{*}{$\begin{array}{l}\text { Date } \\
(1990)\end{array}$} & \multicolumn{2}{|l|}{ DDP } & \multicolumn{3}{|c|}{ RANN-DDP } & \multicolumn{3}{|c|}{ KANN-DDP ( 5 nodes) } & \multicolumn{3}{|c|}{ KANN-DDP ( 9 nodes) } \\
\hline & $\begin{array}{l}\text { CPU } \\
\text { time } \\
\text { (s) }\end{array}$ & $\begin{array}{l}\text { Cost } \\
\text { (NT\$) }\end{array}$ & $\begin{array}{l}\text { CPU } \\
\text { time } \\
\text { (s) }\end{array}$ & $\begin{array}{l}\text { Cost } \\
\text { (NT\$) }\end{array}$ & $\begin{array}{l}\text { Difference }^{\mathrm{a}} \\
(\%)\end{array}$ & $\begin{array}{l}\text { CPU } \\
\text { time } \\
\text { (s) }\end{array}$ & $\begin{array}{l}\text { Cost } \\
\text { (NT\$) }\end{array}$ & $\begin{array}{l}\text { Difference } \\
(\%)\end{array}$ & $\begin{array}{l}\mathrm{CPU} \\
\text { time } \\
(\mathrm{s})\end{array}$ & $\begin{array}{l}\text { Cost } \\
\text { (NT\$) }\end{array}$ & $\begin{array}{l}\text { Difference } \\
\text { (1/ii) }\end{array}$ \\
\hline $\begin{array}{l}1 \text { June } \\
\text { (Fri.) }\end{array}$ & 148.01 & 159146592 & 20.94 & 159148600 & 0.00126 & 25.65 & 159151200 & 0.00290 & 25.88 & 159150896 & 0.00270 \\
\hline $\begin{array}{l}1 \text { July } \\
\text { (Sun.) }\end{array}$ & 133.01 & 123179936 & 13.29 & 123180144 & 0.00017 & 11.95 & 123179880 & 0.00005 & 16.81 & 123185472 & 0.00449 \\
\hline $\begin{array}{l}1 \text { Aug. } \\
\text { (Wed.) }\end{array}$ & 159.54 & 168468384 & 17.26 & 168475816 & 0.0041 & 21.32 & 168475024 & 0.00394 & 23.08 & 168474904 & 0.00387 \\
\hline
\end{tabular}

${ }^{\text {a Difference }}=\frac{\mid \text { cost of proposed approach }- \text { cost of DDP approach } \mid}{\text { cost of DDP approach }} \times 100 \%$

hydro plants over the 24-hour scheduling period. Details of the water released for the other plants are not given due to limited space.

\subsection{Results using Kohonen's neural network}

The 89 load records were used to train the Kononen net. After 800 iterations of presentations, the neural net mapped these patterns onto the $11 \times 11$ grid as shown in Fig. 10. The digits associated with each node in Fig. 10 indicate the number of input patterns mapped to that node. By using the trained net with its weight connections, we can map the three reserved vectors onto the output nodes. Thus, for each vector with its mapped node, we can find similar patterns within the 89 records by collecting those records which are mapped onto the same output node or four neighboring nodes. Thus all load patterns which are mapped to the five nodes are said to be of the same cluster. For example, the records of 1 August 1990 mapped to node $(1,2)$. Thus all

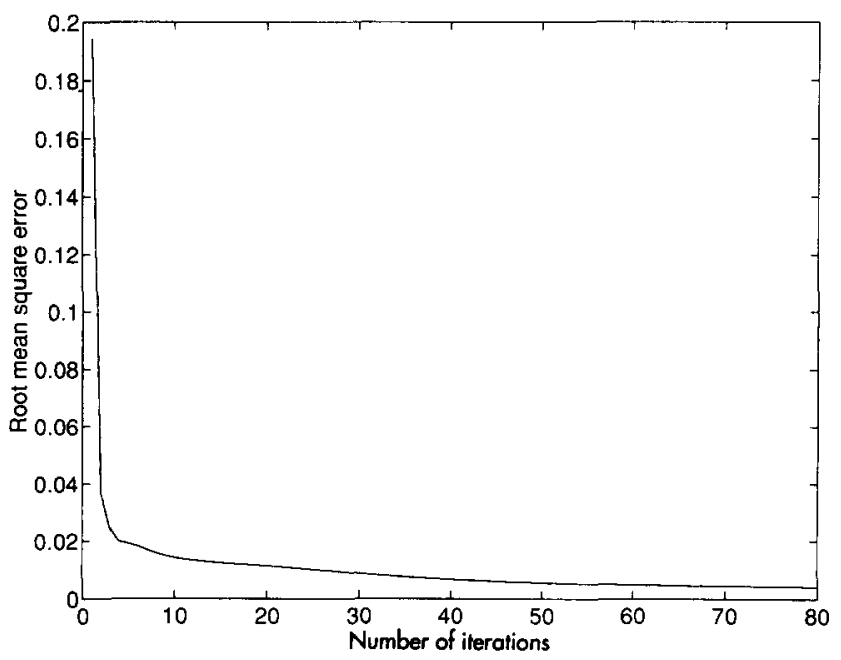

Fig. 8. The r.m.s. errors at each iteration. records which mapped to nodes $(1,2),(1,1),(1,3)$, $(2,2)$ and $(11,2)$ were defined to be of the same cluster. The preliminary schedule of a load pattern is obtained by averaging the schedule vectors of the load pattern in the cluster. With the preliminary schedule at hand, the final schedule is obtained by using the DDP algorithm.

For the same trained network, we have the freedom to choose the number of neighboring nodes which define a cluster. To examine how the number of neighboring nodes in a cluster affects the final results, we consider another case of eight neighboring nodes. For example, nodes $(1,1),(1,2),(1,3),(2,1),(2,2),(2,3),(11,1)$, $(11,2)$ and $(11,3)$ are selected for the pattern of 1 August 1990. It is obvious that more patterns will be included in a cluster if we select more neighboring nodes. Note that overlapping of clusters is allowed. The results are summarized in Table 2. Fig. 11 illustrates the water release schedules for Te-Chi hydro plant over the 24-hour scheduling period.

\subsection{Discussion}

Based on the results in Table 2, Fig. 9, and Fig. 11, the following observations can be made.

(i) It is observed from the results in Table 2 that the fuel costs for the schedules from the ANN-DDP approach are very close to those for the schedules from the DDP algorithm. However, the ANN-DDP approach requires much less computer time than the DDP algorithm.

(ii) It is found from the results in Figs. 9 and 11 that the final water release schedule for Te-Chi hydro plant reached by the proposed ANN-DDP approach is very similar to that obtained by the DDP algorithm.

(iii) It is also observed from the results in Table 2 that, for the case of 1 July 1990, the 5-node KANNDDP approach gives a schedule with lower costs than that of the DDP. Thus, the ANN-DDP approach may yield a true optimal or near-optimal solution for certain 


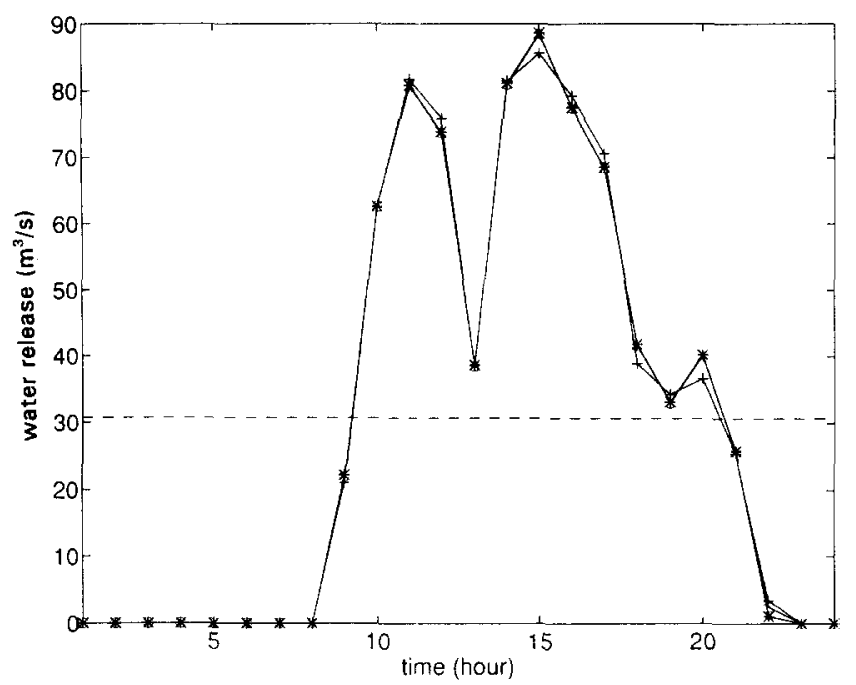

Fig. 9. Water release for Te-Chi hydro plant from DDP (---, initial controls; *, final schedule) and RANN-DDP ( + , preschedule; $O$. final schedule) for the case of 1 June 1990 (Fri.).

load patterns. The corresponding results from the DDP may represent a suboptimal solution due to an improper selection of parameters such as the initial controls.

(iv) By comparing the results of the two ANN approaches, it is observed that the preschedules from the multilayer feedforward ANN are closer to those from the DDP than those from the KANN. This is the main reason why the RANN-DDP requires less computer time than the KANN-DDP in most cases under study.

(v) The training time of the multilayer feedforward ANN is much longer than that of the KANN. In addition, as the scale of the problem is increased, the multilayer feedforward ANN will be even weaker. However, Kohonen's ANN is relatively insensitive to the problem's scale. As far as the training time is concerned, the time spent by the KANN to cluster 89 patterns was about 20 minutes. The training time of the multilayer feedforward ANN with back-propagation training was more than 24 hours in this work.

(vi) We demonstrate the proposed method by using ten hydro plants. It is not difficult to employ a cluster-

\begin{tabular}{|c|c|c|c|c|c|c|c|c|c|c|c|}
\hline & 1 & 2 & 3 & 4 & 5 & 6 & 7 & 8 & 9 & 10 & 11 \\
\hline 1 & 3 & 2 & 2 & 1 & 1 & 1 & 0 & 1 & 0 & 1 & 0 \\
\hline 2 & 1 & 0 & 1 & 0 & 1 & 2 & 0 & 1 & 0 & 0 & 0 \\
\hline 3 & 1 & 1 & 1 & 1 & 0 & 0 & 1 & 0 & 1 & 1 & 2 \\
\hline 4 & 0 & 0 & 1 & 0 & 2 & 1 & 2 & 2 & 1 & 1 & 1 \\
\hline 5 & 2 & 0 & 0 & 2 & 1 & 0 & 4 & 1 & 1 & 2 & I \\
\hline 6 & 0 & 1 & 1 & 0 & 0 & 0 & 2 & 2 & 1 & 0 & 0 \\
\hline 7 & 1 & 1 & 0 & 0 & 3 & 0 & 0 & 0 & 0 & 0 & 0 \\
\hline 8 & 1 & 1 & 0 & 0 & 1 & 2 & 1 & 2 & 0 & 0 & 1 \\
\hline 9 & 0 & 0 & 1 & 1 & 0 & 0 & 0 & 0 & 0 & 2 & 0 \\
\hline 10 & 1 & 1 & 1 & 1 & 0 & 1 & 3 & 0 & 1 & 0 & 0 \\
\hline 11 & 0 & 0 & 0 & 0 & 0 & 0 & 0 & 0 & 1 & 1 & 1 \\
\hline
\end{tabular}

Fig. 10. The mapped net of the 89 patterns.

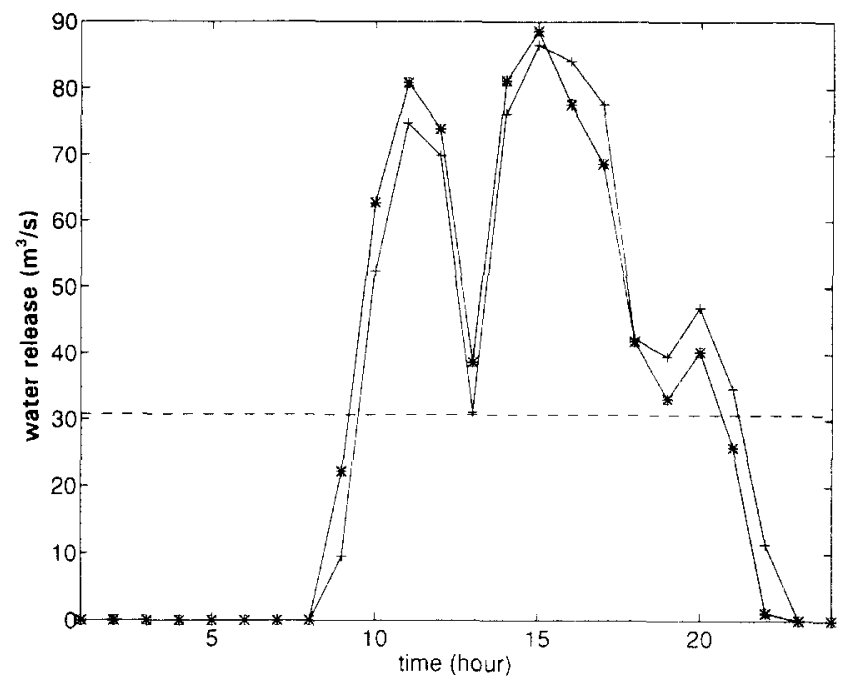

Fig. 11. Water release for Te-Chi hydro plant from DDP (---, initial controls; *, final schedule) and a nine-node KANN-DDP $(+$, preschedule; $O$, final schedule) for the case of 1 June 1990 (Fri.).

ing ANN approach on a larger scale and for more complex problems. Basically, clustering ANNs are insensitive to the scale of problems.

\section{Conclusions}

A novel technique using a hybrid artificial neural network-differential dynamic programming approach has been presented in this paper to reach the desired water release schedules for saving the fuel cost of thermal units. Offline calculations are first executed for historical records of load curves and preliminary schedules by using two ANN algorithms are then obtained. In the multilayer feedforward ANN, the hourly loads are taken as its input and a preliminary water release schedule is generated as the inputs. In the Kohonen clustering ANN, the load patterns are classified into groups. In each group, a preschedule is obtained by averaging the schedules in the group. Finally, the preschedule is used as the initial controls of the DDP algorithm to reach the final schedule. Because the preschedule is very close to the final schedule, the execution time of the DPP algorithm can be significantly reduced.

To demonstrate the effectiveness of the proposed method, hydroelectric generation scheduling of Taiwan power system was performed. It is concluded from the results that good hydro generation schedules can be obtained in a very efficient manner by using the proposed ANN-DDP approach.

\section{References}

[1] M.F. Carvalho and S. Soares, An efficient hydro-thermal scheduling algorithm, IEEE Trans. Power Syst., PWRS-2 (1987) $537-542$. 
[2] A.J. Wood and B.F. Wollenberg, Power Generation, Operation and Control, Wiley, New York, 1984.

[3] H. Habibollazadeh and J.A. Bubenko, Application of decomposition techniques to short-term operation planning of hydrothermal power system, IEEE Trans. Power Syst., PWRS-1 (1986) 44-47.

[4] E.B. Heinsson, Optimal short-term operation of a purely hydroelectric system, IEEE Trans. Power Syst., 3(1988) 1072-1077.

[5] S.M. Amado and C.C. Ribeiro, Short-term generation scheduling of hydraulic multi-reservoir multi-area interconnected systems, IEEE Trans. Power Syst., PWRS-2 (1987) 758-763.

[6] D.M. Murray and S. Yakowitz, Constrained differential dynamic programming and its application to multireservoir control, Water Resour. Res., 15 (1979) 1017-1027.

[7] S. Yakowitz, Dynamic programming applications in water resource, Water Resour. Res., 18 (1982) 673-696.

[8] Z. Ouyang and S.M. Shahidehpour, A hybrid artificial neural network-dynamic programming approach to unit commitment, IEEE Trans. Power Syst., 7 (1992) 236-246.

[9] C.H. Chen, S.C. Chang and I.K. Fong, An effective differential dynamic programming algorithm for constrained optimal control problems, Proc. American Control Conf., 1989, pp. 17631764.

[10] D.E. Rumelhart, G.E. Hinton and R.J. Williams, Learning internal representations by error propagation, in D.E. Rumelhart and J.C. McClelland (eds.), Parallel Distributed Processing, Vol. 1, Foundations, MIT Press, Cambridge, MA, 1986, pp. 318-362.

[11] R.P. Lippmann, An introduction to computing with neural nets, IEEE ASSP Mag., 4 (2) (1987) 4-22.
[12] T. Kohonen, Self-Organization and Associative Memory, Springer, Berlin, 3rd edn., 1989

[13] Y.Y. Hsu and C.C. Yang, Design of artificial networks for short-term load forecasting, Parts I and II, IEE Proc. C, 138 (1991) 407-418.

[14] Y.Y. Hsu and C.R. Chen, Tuning of power system stabilizers using an artificial neural network, IEEE Trans. Energy Convers., EC-6 (1991) 612-619.

[15] N.I. Santoso and O.T. Tan, Neural-net based real-time control of capacitors installed on distribution systems, IEEE Trans. Power Delivery, 5 (1990) 266-272.

[16] Z. Ouyang and S.M. Shahidehpour, A multi-stage intelligent system for unit commitment, IEEE Trans. Power Syst., 7 (1992) $639-645$

[17] Y.Y. Hsu and L.H. Jeng, Analysis of torsional oscillations using an artificial neural network, IEEE Trans. Energy Convers., 7 (1992) 684-690.

[18] D.J. Sobajic and Y.H. Pao, Artificial neural-net based dynamic security assessment for electric power systems, IEEE Trans. Power Syst., 4 (1989) 220-228.

[19] H. Mori, Y. Tamaru and S. Tsuzuki, An artificial neuralnet based technique for power system dynamic stability with the Kohonen model, IEEE Trans. Power Syst., 7 (1992) 856864.

[20] D. Neibur and A.J. Germond, Power system static security assessment using the Kohonen neural network classifier, IEEE Trans. Power Syst., 7 (1992) 865-872.

[21] S. Yakowitz and B. Rutherford, Computational aspects of discrete-time optimal control, Appl. Math. Comput., 15 (1984) $29-45$. 